\title{
Nano Factory Achieved by Focused Ion Beam
}

Toshiaki Fujii, and Takashi Kaito*

*Engineering division, SII NanoTechnology Inc., 36-1, Takenoshita, Oyama-cho, Sunto-gun, Shizuoka 410-1393, Japan

For the micro machine fabrication, the MEMS technology is one of the standard technologies currently being used. The MEMS technology is suitable for mass production of very small parts but has a limitation in the fabricable shape due to its etching-based processing method. The FIB system can be used to make the parts having various shapes that cannot be made by the MEMS, so we are now developing a new fabrication technology allowing the incorporation of FIB-made parts into the MEMS-made parts, in order to realize new mechanisms and functions.

\section{3D deposition using 3D-CAD data}

It has been reported that the three-dimensional structures are fabricated by FIB deposition [1]. This report shows that the FIB can be used to fabricate "overhanging shape" such as wine glass, which cannot be made by MEMS, by changing Lissajou curve produced by two deflecting signals with time. To make more complex shapes, we developed a new method using 3D-CAD data. Large variety of 3D shapes are fabricated by slicing a 3D-CAD designed structure into a number of data sheets, depositing the bottom layer of the sliced data at first, and then accumulating all sheets from the bottom in order (Fig. 1).

2. Nano Lathe having precision wheel stage Although the 3D deposition technology allows you to fabricate the shapes that cannot be made by MEMS, the usable material is limited. We developed a stage, which is used in the vacuum chamber of FIB system and rotates with a high degree of accuracy (radial run out of $0.14 \mu \mathrm{m}$ ), achieving a lathe function (Fig. 2) [2]. We can make an intended shape by the irradiation of rotating but non-scanning ion beam to the specimen attached to the precision wheel stage (Fig. 3). Fabrication using nano lathe is a non-material dependant, since the effect of redeposition can be reduced without using GAE by rotating machining surface continuously.

3. Nano tweezers for handling nanostructure fabrication

Combining the parts made by FIB with those made by MEMS will drive the development of micro machines. Currently, we are developing Nano tweezers with Aoi Electronics Co., Ltd. that can be used to handle these small mechanical parts. We also developing an actuator used to move the nano tweezers ends to $\mathrm{X}, \mathrm{Y}$ and $\mathrm{Z}$ directions and to rotate them (Fig. 4). Combining this nano tweezers with the precision wheel stage realizes more flexible assembly.

We are going to achieve the "Nano Factory" which cannot be realized by existing technologies. In the Nano Factory, components are fabricated with FIB system, handled with using a high-resolution microscope observation, and assembled to make micro machines.

\section{References}

[1] Matsui, et al; "Three-dimensional nanostructure fabrication by focused-ion-beam chemical vapor deposition" JVST B, Vol.18, No. 6, Nov./Dec. 2000, pp.3181-3184

[2] Iwasaki, et al., "Nano Factory by Focused Ion Beam”, 4th IWMF, pp.748-753, Oct. 2004 

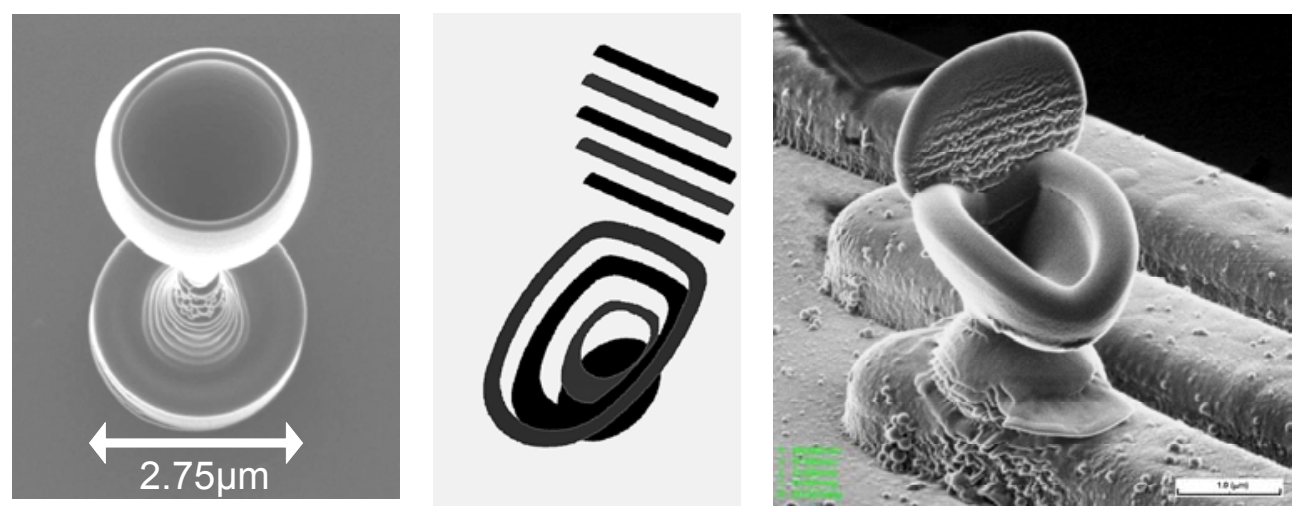

Fig. 1. Wine glass made by deposition (collaborative project with NEC \& Univ. of Hyogo) (left), sliced 3D-CAD data (middle) and nano-toilet (right).
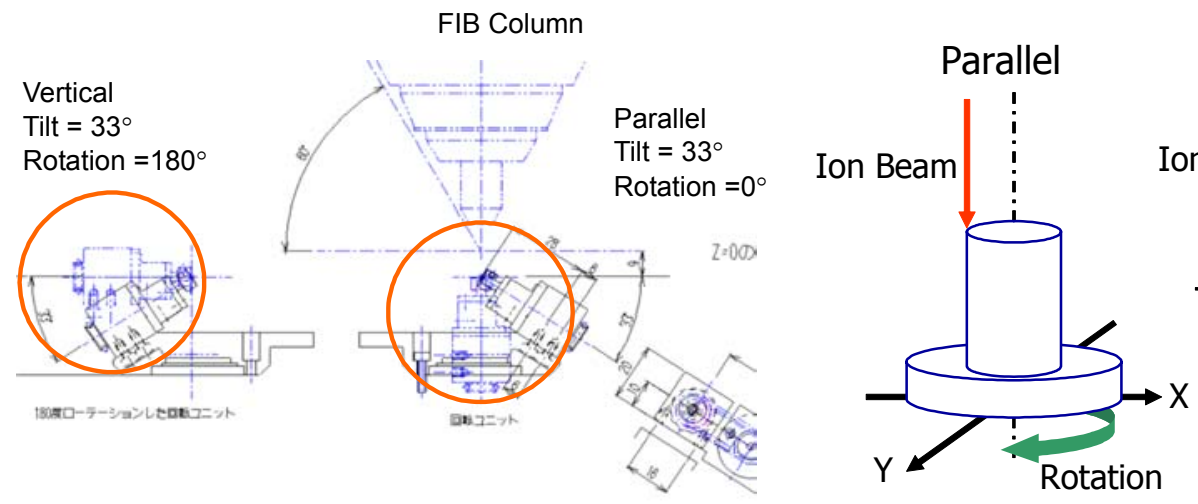

Vertical

Fig. 2. Precision wheel stage (left) and the concept of nano lathe (right)
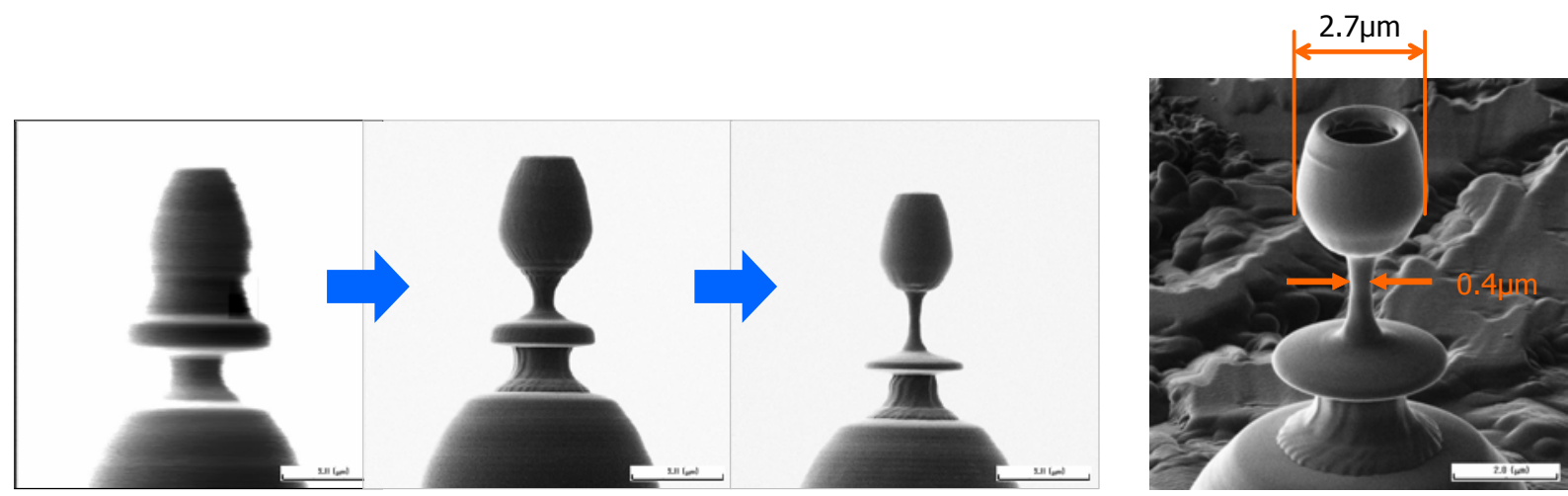

Fig. 3. Nano wing glass processed by nano lathe at $120 \mathrm{rpm}$. Material = carbon.
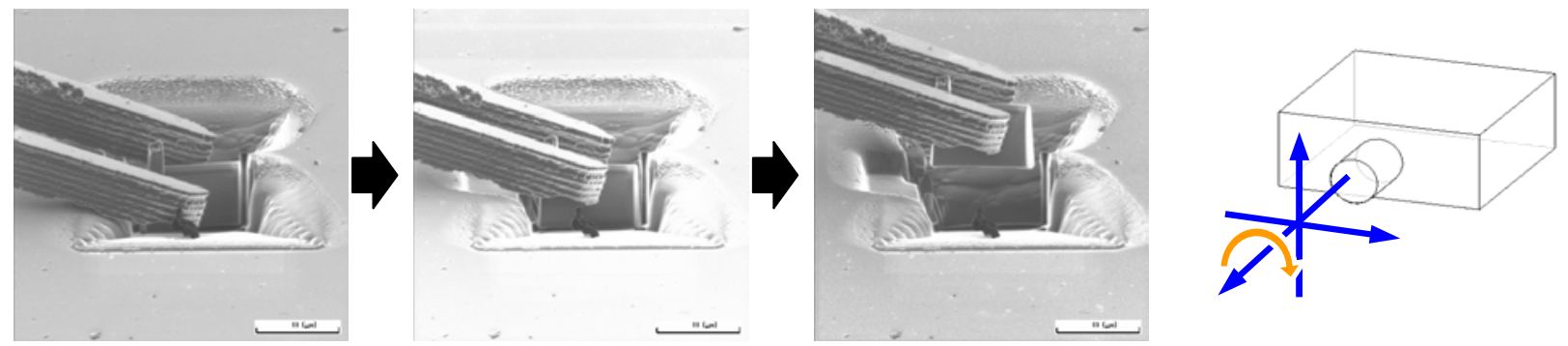

Fig. 4. Nano tweezers (left) and its actuator (right) 